\title{
$\operatorname{tm}$
}

\section{Technisches Messen}

\section{Aufsätze $\cdot$ Papers}

Transiente Anregungen von mechanischen Schwingungen

H. G. Natke

in der Versuchstechnik

Transient excitations of mechanical vibrations

in testing techniques

Digitale Signalverarbeitung in der Schwingungsmeßtechnik

J. Zaschel

Digital signal processing in vibration analysis

Meßsignalverarbeitung mit hochauflösender AD-Wandlung

A. Klauer

Processing measurement signals with high resolution AD-conversion

M. Pandit

Automatische Überwachung des Stanzvorganges

F. Oppel

W. Ecker

durch Auswertung prozeßbedingten Körperschalls

Automatic control of punch processes by using

structure born sound

Kanalkompensation bei der akustischen Güteprüfung

D. Becker

Frequency characteristic compensation within the acoustical quality control

Rechnergesteuerter Meßplatz zur automatischen Ermittlung

H. Lautenschläger

der dielektrischen Festigkeit von Isoliergasen

bei Stoßspannungsbeanspruchung

Microcomputer controled measuring set-up for the

automatic determination of the dielectric strength

of insulating gases under impulse voltage stress

\section{tm-Gastvorlesung $\cdot$ tm Tutorial}

Meßtechnik und Meßsignalverarbeitung

H.-R. Tränkler

Measurement techniques and signal processing

Produktinformationen - Product information 


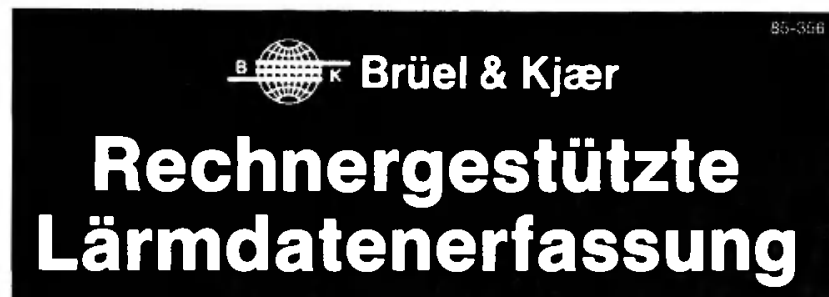

Langzeitüberwachung der Lärmemissionen und -immissionen nach allen relevanten Normen, MeB- und Auswertevorschritten unter schwierigsten Umweltbedingungen in allen Lärmumgebungen, als da sind: Flughäten, Verkehrswege, Industrieanlagen, Grobbaustellen, Kraftwerke, Rallinerien, Werkhallen u.a.

Brujel\& Kjar hat es...

...das kompromiBlose Mebsystem:

tlexibel, präzise, bewährt, normgerecht und in zukunt eichtahig

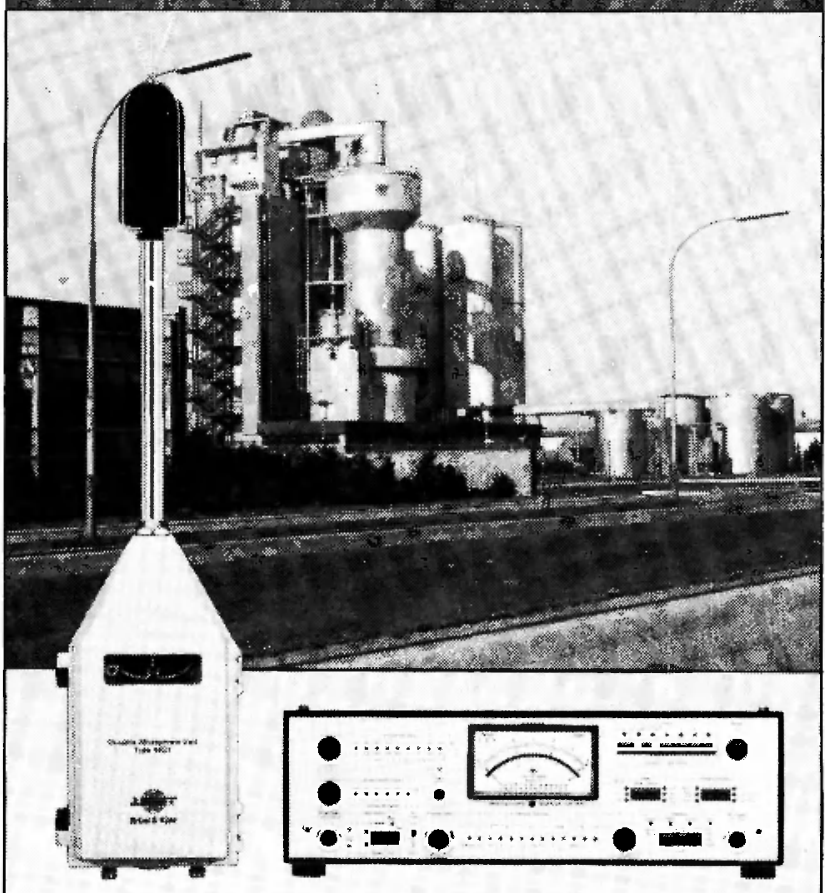

Wetterfeste Mikrofoneinheit Typ 4921 und MeBverstärker Type 2636 bilden zusammen einen Präzisions-Schallpegelmesser (DIN IEC 651, Klasse 1I) mit allen gebräuchlichen Frequenz- und Zeitbewertungen, mit Analoganzeige und Digitalausgang via IEC-Bus-Schnittstelle. Ein angeschlossener IECBus-kompatibler Tischrechner kann alle Funktionen setzen und abfragen, die MeBdaten übernehmen, zu Lărm-Kenndaten und -Beurteilungsgrößen weiterverarbeiten, ablegen und dokumentieren Software nach Wunsch auf Vereinbarung

Fordern Sie ausführliche Unterlagen an !

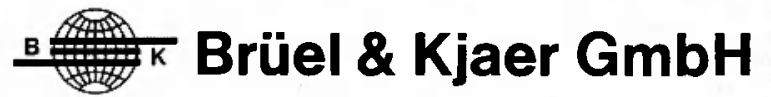

Postfach 1160. 2085 Quickborn. Telefon (04106) 4055 Telex 215084 . Telefax(04 106)69955

Zweigstellen: Düsseldorf (0211)62 7064. Frankfurt (06152) 56374 München (089) 7930944 . Stuttgart (07195) 4548
Technisches Messen - tm

Begründet von Professor Dr.-Ing. Georg Keinath.

Organ der NAMUR (Normenarbeitsgemeinschaft für MeB- und Rcgeltechnik in der Chemischen Industrie).

Mit Mitteilungen der VDI / VDE-Gesellschaft Meß- und Regelungstechnik und der AMA-Arbeitsgemeinschaft Meßwertaufnehmer e.V.

Herausgeber:

Prof. Dr. Joachim Hesse (verantwortlich)

Fraunhofer-Institut für Physikalische Meßtechnik, Heidenhofstraße 8, D-7800 Freiburg.

Wissenschaftlicher Beirat:

Dr.-Ing. R. Best, Basel,

Dipl.-Phys. W. Boehme, Karlsruhe,

Prof. Dr. T. Gast, Berlin,

Dipl.-Phys. A. Happe, Wolfsburg,

Dipl.-Ing. K. Heitmann, Wetzlar,

Dir. G. Klee, Frankfurt,

Dr. J. König, Leverkusen,

Dr.-Ing. N. Korn, Frankfurt,

Prof. Dr. F. Mesch, Karlsruhe,

Dipl.-Ing. E. Sennhenn, Darmstadt,

Dr. B. Will, Frankfurt.

\section{Verlag:}

R. Oldenbourg Verlag GmbH, Rosenheimer Straße 145 ,

D-8000 München 80, Telefon (089)41 12-0, Telex 5-29296 (rover), Telefax-Nr. (089) 4112207.

Redaktionsbüro im Verlag:

Therese Gicklhorn, Telefon (089) 4112221.

Zusendungen von Originalbeiträgen werden an den Herausgeber erbeten. Für unverlangt eingesandte Manuskripte wird keine Haftung übernommen.

\section{Anzeigenverwaltung:}

R. Oldenbourg Verlag GmbH.

Verantwortlich für den Anzeigenteil: Konrad Haslbeck, Telefon (089) 4112223 , Anschrift siehe Verlag.

Zur Zeil gilt Anzeigenpreisliste Nr. 34.

\section{Bezugsbedingungen:}

"Technisches Messen - tm" erscheint monatlich einmal.

Jahresahonnementspreis Inland DM 136,- DM 12,- Versandspesen $=$ DM 148,- (incl. 7,0\% MwSt = DM 9,68). Ausland DM 136,- + DM 14,40 Versandspesen $=$ DM 150,40

Einzelheft Inland DM 20,- + DM 1,- Versandspesen = DM 21,(incl. 7,0\% MwSt $=$ DM 1,37), Ausland DM 20,- + DM 1,20 Versandspesen

Studierende erhalten auf den Jahresbezugspreis von DM 136,- eine Ermäßigung von $2 \overline{0} \%$.

Bestellungen können über jede Buchhandlung aufgegeben oder direkt an den Verlag gerichtet werden. Abonnements-Kündigung 8 Wochen zum Ende des Kalenderjahres. - Jahres-Inhaltsverzeichnis im Dezemberheft

Die in dieser Zeitschrift veröffentlichten Beiträge sind urheberrechtlich geschützt. Alle Rechte, auch das der Übersetzung in fremde Sprachen, bleiben vorbehalten. Kein Teil dieser Zeitschrift darf ohne schriftliche Genehmigung des Verlages in irgendeiner Form - durch Fotokopie, Mikrofilm oder andere Verfahren - reproduziert oder in eine von Maschinen, insbesondere von Datenverarbeitungsanlagen, verwendbare Sprache übertragen werden. Auch die Rechte der Wiedergabe durch Vortrag, Funk- und Fernsehsendung, im Magnettonverfahren oder ähnlichem Wege bleiben vorbehalten.

tonverfahren oder ahnlichem Wege bleiben vorbehalte Fotokopien für den persönlichen und sonstigen eigenen Gebrauch dūrfen nur von einzelnen
Beiträgen oder Teilen daraus als Einzelkopien hergestellt werden. Jede im Bereich eines gewerblichen Unternehmens hergestellte oder benützte Kopie dient gewerblichen Zwecken gem. \$54 (2) UrhG und verpflichtet zur Gebührenzahlung an die VG Wort, Abteilung Wissenschaft, Goethestraße 49, 8000 München 2, von der die einzelnen Zahlungsmodalitäten zu erfragen sind. (Zeitungsvertriebsamt für die Deutsche Demokratische Republik: DDR-1004 Berlin, Straße der Pariser Kommune 3-4.) 